\title{
Balanced Scorecard em instituições de ensino: aplicação em um Instituto Federal de Santa Catarina
}

Planejamento e gestão estratégica estão cada vez mais presentes no cotidiano das organizações públicas, baseados principalmente em experiências do setor privado. Neste sentido, a instituição objeto deste estudo tornou público, em 30 de dezembro de 2014 , a primeira edição do seu planejamento estratégico, o Planejamento Estratégico 2013-2017. No entanto, no planejamento não estavam previstos mecanismos para avaliar o desempenho da instituição. Assim, o objetivo deste trabalho é aplicar a ferramenta de avaliação de desempenho Balanced Scorecard (BSC) em um Instituto Federal localizado no estado de Santa Catarina. Este estudo é uma pesquisa-ação com objetivo descritivo, no qual a definição do portfólio bibliográfico ocorreu com a utilização da metodologia Proknow-C, bem como foram utilizados livros e outros artigos relacionados ao tema. Partindo dos objetivos estratégicos pré-elaborados no planejamento estratégico da instituição e após entrevistas com os gestores, elaborou-se o painel de desempenho do BSC que dará subsídio para os gestores acompanharem a realização dos objetivos estratégicos em relação às metas propostas. Por meio do estudo, observou-se que o BSC pode ser considerado como uma promissora ferramenta para a avaliação de desempenho de instituições de ensino e constatou-se que dos sete objetivos estratégicos analisados, apenas o objetivo estratégico "criar manuais e fluxos de trabalho no IFC" não havia atingido 50\% da meta, que é implantar até o final de 2017.

Palavras-chave: Avaliação de Desempenho; Balanced Scorecard; Instituições de Ensino.

\section{Balanced Scorecard in teaching institutions: application at a Federal Institute of Santa Catarina}

Strategic planning and management are increasingly present in the day-to-day of public organizations, mainly based on private sector experiences. In this sense, the institution object of this study made public, on December 30,2014, the first edition of its strategic planning, Strategic Planning 2013-2017. However, in the planning, mechanisms were not foreseen to evaluate the performance of the institution. Thus, the objective of this work is to apply the Balanced Scorecard (BSC) performance evaluation tool to a Federal Institute located in the state of Santa Catarina. This study is an action research with a descriptive purpose, in which the definition of the bibliographic portfolio occurred with the use of the Proknow-C methodology, as well as the use of books and other articles related to the subject. Based on the strategic objectives pre-elaborated in the strategic planning of the institution and after interviews with the managers, the performance panel of the BSC was elaborated, which will give support to the managers to follow the achievement of the strategic objectives in relation to the proposed goals. Through the study, it was observed that the BSC can be considered as a promising tool for evaluating the performance of educational institutions and it was verified that of the seven strategic objectives analyzed, only the strategic objective "to create manuals and workflows in the IFC "had not met $50 \%$ of the target, which is to implement until the end of 2017.

Keywords: Performance Measurement; Balanced Scorecard; Teaching Institution.

\section{Topic: Gestão Pública}

Reviewed anonymously in the process of blind peer
Received: 15/10/2017

Approved: 17/12/2017

Mariana da Silveira

Universidade Federal de Santa Catarina, Brasil

http://lattes.cnpq.br/8145003675490174

mariana.dasilveira1@gmail.com

Sergio Murilo Petri

Universidade Federal de Santa Catarina, Brasil

http://lattes.cnpq.br/2579064028361832

smpetri@gmail.com

Referencing this:

SILVEIRA, M.; PETRI, S. M.. Balanced Scorecard em instituições de ensino: aplicação em um Instituto Federal de Santa Catarina. Revista Brasileira de Administração Científica, v.8, n.3, p.122-134, 2017. DOI: http://doi.org/10.6008/SPC2179-684X.2017.003.0010 


\section{INTRODUÇÃO}

Os Institutos Federais de Educação, Ciência e Tecnologia foram criados por meio da Lei no 11.892, de 29 de dezembro de 2008, e integram a Rede Federal de Educação Profissional e Tecnológica. Com a recente expansão da Rede Federal, que teve 214 instituições entregues entre 2003 e 2010 (BRASIL, 2016), e com o reconhecimento e a visibilidade alcançados, aumentaram as cobranças, tanto do governo quanto da sociedade, e a necessidade de planejar suas ações institucionais para direcionar os rumos das Instituições nos próximos anos.

Waweru et al. (2007) corrobora citando que as duas últimas décadas testemunharam uma crescente pressão sobre as organizações do setor público para se tornarem mais eficientes e eficazes, o que necessitou de uma grande melhoria nos seus sistemas de custos e de gestão, de modo a proporcionar informações de qualidade e oportunas.

Neste sentido, a instituição objeto deste estudo formou no final de 2012 uma equipe organizadora para elaborar a primeira edição do seu planejamento estratégico e, em 30 de dezembro de 2014, tornou público o Planejamento Estratégico 2013-2017. No planejamento definiu-se a estratégia global do instituto para os cinco anos seguintes, representada por um conjunto de objetivos estratégicos que descrevem como a instituição pretende cumprir sua missão institucional e alcançar sua visão de futuro, direcionando seu comportamento e desempenho (IFC, 2014).

Bryson (2003, citado por PUீČEK et al., 2014) afirma que planejamento e gestão estratégica estão cada vez mais parte da vida das organizações do setor público em todo o mundo, baseada principalmente em experiências do setor privado. No entanto, para obter sucesso, é importante fazer um acompanhamento contínuo do plano. Em um estudo sobre o impacto da avaliação de desempenho no planejamento estratégico, Tapinos et al. (2005) relata que a avaliação de desempenho revelou ter influência significativa no apoio à consecução dos objetivos de uma organização e da eficácia e eficiência de seu processo de planejamento estratégico.

Uma das ferramentas de avaliação de desempenho que é amplamente utilizada por empresas do setor privado e possui recentes estudos sobre sua aplicação em instituições de ensino públicas e privadas é o Balance Scorecard (BSC) (PAPENHAUSEN et al., 2006; UMASHANKAR et al., 2007; PHILBIN, 2011; WU et al., 2011; ZANGOUEINEZHAD et al., 2011; SCHOBEL et al., 2012; SAYED, 2013; AL-HOSAINI et al., 2015; HLADCHENKO, 2015).

Desenvolvido por Kaplan et al. (1992), o BSC busca alinhar o planejamento estratégico com as ações operacionais da empresa. Deste modo, considerando o pressuposto do BSC como ferramenta de avaliação de desempenho, o problema a ser solucionado pela presente pesquisa consiste em: Como seria estruturado o painel estratégico de um Instituto Federal de Educação utilizando o modelo do Balanced Scorecard?.

Dentre as razões que justificam a realização deste estudo, destacam-se a realização do acompanhamento do planejamento estratégico do instituto para verificar a atual situação das metas propostas, e a colaboração com a literatura na área de avaliação de desempenho utilizando Balance Scorecard em instituições públicas de ensino. 
A estrutura do presente estudo é composta pela introdução; referencial teórico, que aborda conceitos sobre planejamento estratégico, avaliação de desempenho e BSC; a metodologia de pesquisa, que apresenta os procedimentos metodológicos utilizados; os resultados; as considerações finais e, por fim, as referências bibliográficas utilizadas.

\section{REVISÃO TEÓRICA}

\section{Planejamento estratégico no setor público}

Nesta seção, serão abordados os conceitos chaves sobre Planejamento Estratégico no Setor Público, Avaliação de Desempenho e BSC com sua aplicação em Instituições de Ensino. Planejar estrategicamente consiste em combinar as oportunidades oferecidas pelo ambiente externo às condições internas da empresa, identificando e destacando os pontos fortes e fazendo com que os pontos fracos não prejudiquem, de modo a satisfazer seus objetivos futuros. Segundo Tapinos et al. (2005), planejamento estratégico é o conjunto de processos adotados visando desenvolver uma série de estratégias que contribuirão para ter êxito na direção organizacional.

As etapas do Planejamento Estratégico, segundo Hitt et al. (2003), podem ser agrupadas em: Concepção estratégica: declaração de missão (a razão de ser da instituição) e visão de negócios (como a empresa pretender ser no futuro); Gestão do conhecimento estratégico: diagnóstico estratégico externo e interno - levantamento das oportunidades, ameaças externas e forças e fraquezas internas - e construção de cenários; Formulação estratégica por meio do sistema de Planejamento Estratégico: elaboração de ações por meio de planos operacionais e definição dos objetivos, que devem seguir uma hierarquia de importância, prioridade ou urgência; e Implementação da estratégia: questões de estrutura e controles organizacionais, governança corporativa e liderança estratégica.

Em relação à administração pública, uma característica específica do planejamento estratégico e gestão estratégica é a necessidade de se adaptar ao ambiente burocrático e a influências políticas, mas isso não significa ignorar os elementos de gestão estratégica (PU゚ČEK et al., 2014). Devido à ausência de competitividade e de outras formas de se mensurar desempenho, a percepção e o senso de utilidade de adoção do Planejamento Estratégico, como usualmente aplicado em ambientes competitivos, podem ser diferentes. Neste sentido, a utilização de Planejamento Estratégico (PE) no setor público é mais recente que na esfera privada, e, nos últimos anos, seu uso vem crescendo gradualmente (PEREIRA, 2006).

Giacobbo (1997), pesquisando sobre a motivação de organizações públicas para implementação de Planejamento Estratégico, apontou como um dos motivos acabar com a descontinuidade administrativa gerada pela troca da administração. Em vários órgãos públicos, a troca de gestão acarreta a falta de continuidade nos projetos em andamento, gerando assim desperdício de dinheiro público. Porém, mesmo com o aumento de sua utilização, ainda existe carência de estudos sobre o processo de formação do Planejamento Estratégico em instituições públicas (GIACOBBO, 1997). 


\section{Avaliação De Desempenho}

Por meio da adoção de um sistema de avaliação de desempenho, é possível fazer um acompanhamento do plano, verificando se os objetivos propostos no planejamento estratégico estão sendo atingidos. A medição do desempenho refere-se ao uso de informações financeiras e não financeiras pelos gestores para tomar decisões relativas às atividades organizacionais com foco nos objetivos prédeterminados (DE WAAL, 2007). Portanto, avaliação de desempenho refere-se ao conjunto de ações postas em prática para mensurar o quanto uma organização está atingindo os seus objetivos pré-determinados (AMARATUNGA et al., 2001).

Em uma pesquisa realizada por Tapinos et al. (2005), onde investigava o impacto da avaliação de desempenho no planejamento estratégico, uma das conclusões mais importantes determinou que a medição de desempenho destaca-se como um dos os fatores-chave para o PE. Para os autores, isto tem um valor duplo, pois, em primeiro lugar, reforça os argumentos que os sistemas de medição de desempenho têm um papel crítico na tradução estratégia em ação (KAPLAN et al., 1996, citado por TAPINOS et al., 2005); e em segundo lugar, ele mostra que a medição de desempenho tem um papel de apoio no desenvolvimento de estratégias (TAPINOS et al., 2005, citado por TAPINOS et al., 2005).

Para Neely (2005), um Sistema de Medição de Desempenho pode perder sua eficácia ao longo do tempo se não for reformulado para melhor atender novas demandas ambientais e organizacionais. A reformulação é um processo básico relacionado à medição de desempenho. Deve ser estabelecido como uma funcionalidade embutida no sistema de gestão estratégica (LIMA et al., 2009).

A necessidade de as organizações alinharem suas estratégias com os seus sistemas de medição de desempenho está bem estabelecido na literatura (DYSON, 2000; MCADAM et al., 2002). Com isso, um grande número de estruturas integradas tem sido desenvolvido como o Balanced Scorecard, o Performance Prism, o Performance Pyramid, o Integrated Performance Measurement System e a Cambridge Performance Measurement Methodology (TAPINOS et al., 2005).

\section{Balanced Scorecard e sua aplicação em instituições de ensino}

Desenvolvido por Kaplan et al. (1992), o Balanced Scorecard (BSC) busca alinhar o planejamento estratégico com as ações operacionais da empresa, ou seja, alinhar todos objetivos da alta administração, diretoria, gerências e chão de fábrica, buscando uma visão abrangente de toda a organização (YU et al., 2009).

O BSC contribui com a implantação da estratégia posicionando-a como o centro organizacional, tirando os aspectos financeiros do topo da hierarquia e colocando-os no mesmo patamar de outras questões pertinentes ao negócio - como a gestão dos processos e o relacionamento da empresa com seus clientes. 0 método complementa as análises financeiras com análises sobre a satisfação do consumidor, participação de mercado, processos internos e atividade de melhoria e inovação das organizações - análises operacionais que norteiam o desempenho financeiro futuro (ROSA et al., 2014). 
Tradicionalmente utilizado por empresas que visam ao lucro, uso do BSC vem se popularizando em outras áreas. A sua aplicação em instituições de ensino superior (IES) públicas e privadas foi tema de diversas pesquisas recentes (PAPENHAUSEN et al., 2006; UMASHANKAR et al., 2007; PHILBIN, 2011; WU et al., 2011; ZANGOUEINEZHAD et al., 2011; SCHOBEL et al., 2012; SAYED, 2013; AL-HOSAINI et al., 2015; HLADCHENKO, 2015). Para Umashankar et al. (2007), a abordagem do BSC oferece às IES a oportunidade de formular uma série de medidas para traduzir a missão de criação, compartilhamento e utilização de conhecimento, em um quadro de mobilização global compreensível e coerente - para usuários internos e externos.

Philbin (2011) relata os benefícios decorrentes da utilização do BSC em IES, que são: Acesso a uma fonte central de dados e informações que anteriormente eram localizados em áreas dispersas em toda a instituição; Os relatórios Scorecard fornecem informações específicas sobre o desenvolvimento da instituição, capacidade de pesquisa e ensino, e estas informações contribuem para uma melhor tomada de decisão, como por exemplo, sobre quais cursos de formação desenvolver no futuro; Identificação do valor monetário de alavancagem financeira, que é utilizado pelos financiadores da instituição para justificar o valor do seu investimento.

Tem-se também, em continuidade aos benefícios, a capacidade de monitorar e medir o desempenho da instituição sistemicamente, que inclui considerações de finanças, desenvolvimento de pessoas, a capacidade da instituição e produção de pesquisas, contribui para a sustentabilidade da instituição mediante a identificação de resultados tangíveis e evidências para apoiar o caso de negócio da instituição.

De acordo com Sayed (2013), o BSC tem um olhar abrangente da organização. Ele garante que o processo de planejamento seja organizado e focado e ocorra como um processo regular. Ele é baseado em uma avaliação realista das capacidades organizacionais. É uma base de dados, lida com fatos e tem todas as vantagens de um sistema de banco de dados, desenvolve ligações de comunicação dentro dos departamentos e assegura consultas internas. A abordagem BSC também é flexível, participativa, longitudinal e estratégica. As universidades também tendem a adotar esta abordagem por causa de sua familiaridade e, portanto, mais fácil aceitação pelas instituições.

No entanto, Sayed (2013) contrapõe relatando que o BSC parece ter vários inconvenientes que o torna um pouco inadequado para universidades. O desenvolvimento dos indicadores do BSC é muitas vezes subjetivo (MOORAJ et al., 1999), com dificuldade para captar conceitos como qualidade de serviço e satisfação dos stakeholders. Assim, a adoção do BSC não só é complexa, mas exige compreensão abrangente da filosofia subjacente. O BSC também exige um programa de treinamento abrangente e bem construído para todos os funcionários (WICKRAMASINGHE et al., 2007), o aumento da necessidade de informação clara, válida e oportuna, um investimento de tempo significativo no desenvolvimento e implementação dos processos e, consequentemente, uma demanda pesada de recursos (GAUTREAU et al., 2001; WALL et al. 2011).

Analisou-se a utilização do BSC em IES, a fim de identificar as perspectivas mais adequadas para avaliar o desempenho das instituições. Embora, as quatro principais perspectivas convencionais sejam: financeira, clientes, processos internos e aprendizado e crescimento, os resultados deste trabalho indicam 
que as universidades e instituições de ensino superior como organizações sem fins lucrativos podem aplicar outras perspectivas não financeiras, tais como a participação da comunidade, inovação, parceria estratégica e excelência da investigação científica.

Para Hladchenko (2015), A aplicação do BSC no processo de gestão estratégica de IES contribui para a transparência da implementação da estratégia em diferentes fases do processo e em diferentes níveis da organização. No entanto, apesar de todas as suas vantagens, o BSC não é amplamente utilizado no processo de gestão estratégica de IES devido ao fato de os gestores das universidades não terem experiência e habilidades no desenvolvimento desta ferramenta.

Apesar de ser difícil e de desenvolvimento demorado, o BSC é uma ferramenta eficiente de gestão estratégica que cria um alinhamento entre todos os elementos da estratégia da IES, fazendo o processo de implementação de fácil percepção para os membros da universidade, transparente e mensurável. As perspectivas do BSC dão a oportunidade de desenvolver um equilíbrio entre todos os processos internos e externos, que são fatores na base da estratégia da IES.

\section{Pesquisas similares}

Apresenta-se, no quadro 1, estudos similares que propuseram a aplicação do Balanced Scorecard em instituições de ensino. Destes trabalhos apresentados no quadro 1, destaca-se o estudo de Farias et al. (2012) por apresentar os objetivos estratégicos e indicadores para perspectiva processos internos para uma Instituição Federal de Ensino. Além deste, ressalta-se o trabalho de Martins (2015), que propôs um mapa estratégico na forma do BSC para uma Universidade Estadual que, de certa forma, apresenta similaridades. A partir do encontrado na literatura que descreve o processo de implantação do BSC em instituições de ensino, deseja-se elaborar um BSC para o Instituto Federal estudado.

Quadro 1: Pesquisas similares.

\begin{tabular}{|c|c|c|c|}
\hline \begin{tabular}{|l} 
Autor \\
(ano) \\
\end{tabular} & Objetivo & Resultado & Instrumento de Pesquisa \\
\hline $\begin{array}{l}\text { Martins } \\
(2015)\end{array}$ & $\begin{array}{l}\text { Apresentar uma proposição de } \\
\text { um mapa estratégico na forma } \\
\text { do Balanced Scorecard para a } \\
\text { Universidade Estadual do } \\
\text { Oeste do Paraná - UNIOESTE. }\end{array}$ & $\begin{array}{l}\text { Proposição de um total de } 05 \text { perspectivas: } \\
\text { Educacional, Financeira, Sociedade, Aprendizado e } \\
\text { Crescimento e Processos Internos, distribuídos nestas } \\
\text { perspectivas, } 16 \text { objetivos estratégicos e } 30 \\
\text { indicadores estratégicos. }\end{array}$ & \begin{tabular}{|l|} 
A coleta de dados foi realizada em agosto/2013, com \\
a análise de dados secundários, a utilização do Plano \\
de Desenvolvimento Institucional (PDI) de 2007, \\
Relatório Anual das Atividades da Unioeste, \\
exercícios de 2007 a 2010, Projeto Político \\
Pedagógico Institucional de 2007, Planejamento \\
Institucional da Unioeste de 2007, Planejamento \\
Estratégico Participativo de 2007, Relatório de \\
Atividades 2012 e organogramas da Unioeste \\
(Reitoria e Campis).
\end{tabular} \\
\hline \begin{tabular}{|l|} 
Ulyssea \\
et al. \\
(2013)
\end{tabular} & $\begin{array}{l}\text { Propor um modelo para a } \\
\text { gestão estratégica } \\
\text { organizacional a uma } \\
\text { Instituição de Ensino Superior, } \\
\text { por meio da estruturação de } \\
\text { um Balanced Scorecard (BSC). }\end{array}$ & \begin{tabular}{|l|} 
Os resultados da pesquisa indicam que: (i) as \\
estratégias da instituição estão traduzidas em \\
iniciativas e ações, por meio da ferramenta BSC; (ii) a \\
instituição traduziu os objetivos estratégicos do Plano \\
de Desenvolvimento Institucional em metas \\
operacionais e (iii) o mapeamento da instituição \\
desenvolvido por meio da metodologia BSC gerou \\
uma visão holística e alinhada das estratégias \\
desdobrando-as até seu nível operacional.
\end{tabular} & $\begin{array}{l}\text { Para a elaboração do trabalho observou-se e } \\
\text { coletou-se informações consideradas de } \\
\text { fundamental importância para a IES, a fim de } \\
\text { estruturar o modelo de ferramenta gerencial, de } \\
\text { acordo com a realidade da organização. Fez-se uso, } \\
\text { ainda, do método de pesquisa observacional. }\end{array}$ \\
\hline $\begin{array}{l}\text { Farias et } \\
\text { al. } \\
(2012)\end{array}$ & $\begin{array}{l}\text { Proposição de um mapa } \\
\text { estratégico como ferramenta } \\
\text { de comunicação da estratégia } \\
\text { no âmbito de uma Instituição } \\
\text { de Ensino Público Federal. }\end{array}$ & $\begin{array}{l}\text { Com relação aos resultados, o mapa proposto se } \\
\text { desdobrou em cinco perspectivas, explicadas por } 17 \\
\text { objetivos estratégicos. Para atender às } \\
\text { especificidades da organização em estudo, foram } \\
\text { realizadas adaptações na perspectiva clientes, } \\
\text { alterada para alunos/sociedade, perspectiva } \\
\text { financeira, substituída pela perspectiva orçamentária }\end{array}$ & $\begin{array}{l}\text { Análise do Planejamento Institucional 2011/2012; } \\
\text { Entrevistas com os servidores ligados ao } \\
\text { Planejamento Institucional, essas entrevistas tiveram } \\
\text { por objetivo obter informações mais completas } \\
\text { acerca do que os envolvidos sabem, acreditam, } \\
\text { sentem e esperam a respeito da situação em estudo. }\end{array}$ \\
\hline
\end{tabular}




\begin{tabular}{|c|c|c|c|}
\hline & & $\begin{array}{l}\text { e foi incluída a perspectiva da responsabilidade } \\
\text { social. }\end{array}$ & \\
\hline $\begin{array}{l}\text { Dalfovo } \\
\text { et al. } \\
(2009)\end{array}$ & $\begin{array}{l}\text { Apresentar um estudo e } \\
\text { viabilidade para Implantação } \\
\text { do BSC em uma Instituição de } \\
\text { Ensino Superior, como um } \\
\text { Observatório da Educação. }\end{array}$ & $\begin{array}{l}\text { Como resultado implantou-se o BSC na IES, e com } \\
\text { isso, pôde-se ter uma visão macro dos indicadores de } \\
\text { desempenho dos cursos de pós-graduação em nível } \\
\text { lato sensu. }\end{array}$ & $\begin{array}{l}\text { O estudo foi realizado em etapas, sendo a primeira } \\
\text { caracterizada pela pesquisa bibliográfica, que } \\
\text { proporcionou aprofundamento na questão teórica } \\
\text { dos métodos de medição de desempenho, em } \\
\text { especial o BSC. Concomitante a este processo } \\
\text { ocorreu a pesquisa documental e descritiva, por } \\
\text { meio do levantamento das ementas das disciplinas } \\
\text { das Pós Graduações analisadas e a montagem de } \\
\text { questionário de avaliação. O questionário aplicado } \\
\text { limitou-se a questões internas da IES, sendo assim, } \\
\text { não foram apresentadas as características, processos } \\
\text { de lançamento e conteúdo. }\end{array}$ \\
\hline $\begin{array}{l}\text { Costa et } \\
\text { al. } \\
(2007)\end{array}$ & $\begin{array}{l}\text { O trabalho teve como objetivo } \\
\text { compreender a aplicabilidade } \\
\text { de uma ferramenta como o } \\
\text { Balanced Scorecard em uma } \\
\text { Organização Estudantil } \\
\text { Universitária, por meio de um } \\
\text { estudo de caso na organização } \\
\text { não-governamental AIESEC. }\end{array}$ & $\begin{array}{l}\text { A aplicação da ferramenta demonstrou-se de crucial } \\
\text { importância para a evolução da organização a partir } \\
\text { do momento que busca adaptar a visão tradicional } \\
\text { para perspectivas inovadoras como: Impacto, } \\
\text { Viabilizadores, Membros, Processos e Parceiros. Além } \\
\text { disso, fornece suporte para a implantação de novos } \\
\text { escritórios, serve como ferramenta de motivação dos } \\
\text { alunos envolvidos e principalmente articula, } \\
\text { comunica e traduz a estratégia desenhada em âmbito } \\
\text { nacional e internacional para os diversos escritórios, } \\
\text { gerando assim coesão entre eles. }\end{array}$ & $\begin{array}{l}\text { Neste estudo foram utilizadas diversas fontes de } \\
\text { informação como, por exemplo, o levantamento de } \\
\text { documentos e arquivos, a realização de entrevistas } \\
\text { com roteiro semiestruturado e a observação } \\
\text { participante por um dos autores no período de } \\
\text { outubro de } 2006 \text { a abril de } 2007 \text {. }\end{array}$ \\
\hline
\end{tabular}

\section{METODOLOGIA}

\section{Caracterização da pesquisa}

Nesta seção é descrita a caracterização da pesquisa, relatados os procedimentos metodológicos utilizados para o desenvolvimento do referencial teórico e os instrumentos para a coleta de dados. Este trabalho pode ser classificado como pesquisa ação, devido à efetiva participação dos pesquisadores, com objetivo de descrever as etapas para elaboração do painel de desempenho no Instituto Federal.

Quanto aos objetivos, a pesquisa é classificada como descritiva, tendo em vista a descrição do processo de aplicação do Balanced Scorecard na instituição objeto de estudo (BEUREN, 2006). Em relação à abordagem do problema, enquadra-se como qualitativa, pois é contextual e interpretativa, captando os dados a partir da visão dos participantes (GRAY, 2012).

\section{Procedimentos para o desenvolvimento do referencial teórico}

O portfólio bibliográfico para a elaboração da fundamentação teórica foi desenvolvido com base na metodologia Knowledge Development Process-Constructivist (Proknow-C), proposto por Ensslin et al. (2007). Assim, na data de 12 de janeiro de 2016, foram realizadas buscas nos bancos de dados Scopus, SienceDirect e Proquest, disponibilizados na base de periódicos da Coordenação de Aperfeiçoamento de Pessoal de Nível Superior (CAPES), de artigos relacionados à área de Ciências Sociais Aplicadas, publicados no período de 2000 a 2016.

Para isso, foram estabelecidos dois eixos de pesquisa, utilizando as combinações de palavras a seguir: Eixo 1: 'Balanced Scorecard', 'performance measurement' e 'public education' e Eixo 2: 'Balanced Scorecard', 'strategic planning' e university. Deste modo, foram encontrados 2.446 artigos em conformidade com os quesitos até aqui estabelecidos.

Após a realização do teste de aderência das palavras-chave, que não trouxe novas palavras, foi realizada a filtragem do banco de artigos quanto à redundância no software de gerenciamento bibliográfico 
Endnote, sendo excluídas 206 publicações duplicadas e publicações em séries, que acabaram sendo importadas mesmo com os filtros estabelecidos nas bases de dados.

Na sequência foram excluídos os artigos cujos títulos não estavam alinhados ao tema da pesquisa, sendo eliminados 2.385 artigos. Em seguida foi realizado o filtro dos artigos quanto ao reconhecimento científico. Para essa pesquisa, foi considerado com reconhecimento científico o artigo com 10 citações ou mais. Com a aplicação deste filtro, obteve-se 33 artigos com reconhecimento confirmado (repositório K) e 28 com reconhecimento ainda não confirmado (repositório P).

Após a leitura de todos os resumos dos artigos do repositório K, foram eliminados 7 artigos que não estavam alinhados, criando o repositório A, e identificados 51 autores, criando o banco de autores. Dos artigos do repositório $\mathrm{P}$, apenas 1 dos artigos que haviam sido publicados há mais de 2 anos estava no banco de autores e 9 eram recentes, publicados a menos de dois anos. Após a leitura destes 10 artigos, verificouse que 8 estavam alinhados com o tema e criou-se o banco de artigos aceitos na reanálise (repositório B).

Com a fusão dos repositórios A e B criou-se o repositório C com 34 artigos. Destes, 9 não estavam disponíveis na íntegra. Foi realizada a leitura dos 25 artigos restantes e constatou-se que 19 estavam alinhados, tendo assim os artigos do Portfólio Bibliográfico. Entretanto, de forma complementar, outros artigos relacionados ao tema e que se enquadravam com o tema desta pesquisa também foram utilizados para agregar valor ao objetivo deste trabalho.

\section{Instrumento para coleta dos dados}

Dos dados utilizados para a aplicação do BSC, os objetivos estratégicos foram retirados do Mapa Estratégico elaborado pela Instituição, conforme Planejamento Estratégico 2013/2017. Para a realização deste estudo, foi utilizada apenas a perspectiva de Processos Internos - Eficiência Operacional. O estudo limitou-se ao Campus Araquari.

A coleta dos demais dados foi realizada por meio de entrevistas com o Coordenador Geral de Administração e Finanças, na ocasião, substituindo o Diretor de Administração Planejamento, com a Diretora de Desenvolvimento Educacional, com o Coordenador Geral de Ensino e com a Coordenadora de Comunicação e Eventos. Foram apresentados os objetivos estratégicos abordados neste estudo para verificar quais as metas e situação atual destes, os indicadores de desempenho, e foi questionado sobre as iniciativas para atingimento das metas.

\section{RESULTADOS E DISCUSSÃO}

Partindo do Mapa Estratégico elaborado como parte do Planejamento Estratégico do IFC, deu-se início a elaboração do painel de desempenho para que se possa acompanhar e mensurar o desempenho do IFC por meio dos indicadores. O Mapa Estratégico do IFC agrupa todas as informações do PE e representa a articulação entre Valores, Missão, Visão e Objetivos Estratégicos em três perspectivas: Atuação Institucional; Processos Internos e Recursos. 
A perspectiva 'Processos Internos', objeto deste estudo, retrata os processos prioritários nos quais o IFC deverá buscar excelência e concentrar esforços a fim de alcançar os objetivos. Os objetivos de Eficiência Operacional estão voltados para o aperfeiçoamento dos processos existentes e para identificação de estratégias que influenciem positivamente nos resultados da instituição (IFC, 2014).

Em relação à construção do Mapa, a estrutura proposta pelos autores para traduzir a estratégia em termos operacionais é, tradicionalmente, dividida em quatro perspectivas: financeira, clientes, processos internos, crescimento e aprendizagem. No entanto, esta estrutura não precisa ser necessariamente desenvolvida de maneira idêntica, sendo possível a adoção de mais ou menos perspectivas com conteúdos mais adequados para organização que está implantando a ferramenta (KAPLAN et al., 1997).

Deste modo, com os objetivos estratégicos pré-determinados pelo $\mathrm{PE}$, partiu-se para a definição dos indicadores de desempenho. Segundo Ferreira et al. (2009), o indicador é uma medida, que pode ser quantitativa ou qualitativa, dotada de significado particular e utilizada para organizar e captar as informações relevantes dos elementos que compõem o objeto da observação. É um recurso que informa empiricamente sobre a evolução do aspecto observado. As definições das metas, da situação atual e das iniciativas foram determinadas conforme a percepção dos gestores do Campus Araquari e da estimativa de realização destes objetivos no Campus, bem como dos meios para que estes objetivos sejam alcançados.

Com a consolidação das informações obtidas por meio das entrevistas, foi elaborado o painel de desempenho da instituição, apresentado no quadro 2, conforme segue: no painel de desempenho, que está disposto em 5 colunas, é possível visualizar os objetivos estratégicos (coluna 1) e os seus correspondentes indicadores de desempenho (coluna 2), a meta da instituição (coluna 3) e sua situação atual (coluna 4), bem como as iniciativas que contribuirão para o alcance da meta (coluna 5).

Ao final da elaboração do painel, é possível verificar a situação atual da realização dos objetivos estratégicos pré-determinados no PE, no âmbito do Campus Araquari. Por meio do painel os gestores poderão fazer o acompanhamento dos objetivos, fazendo ajustes nas iniciativas caso as metas não estejam sendo atingidas.

O BSC enaltece a necessidade dos colaboradores, dos níveis tático e operacional, saberem com clareza quais são os indicadores financeiros e não financeiros para boa execução de suas rotinas diárias e para que os de nível estratégico possam compreender o resultado de suas decisões de longo prazo; e para que se consiga identificar e alinhar os fatores críticos do sucesso organizacional que apontam para o caminho mais curto para o cumprimento da estratégia de longo prazo (KAPLAN et al., 1997).

Quadro 2: Painel de Desempenho.

\begin{tabular}{|l|l|l|l|l|}
\hline Objetivos estratégicos & Indicadores & Metas & $\begin{array}{l}\text { Situação } \\
\text { atual }\end{array}$ & Iniciativas \\
\hline $\begin{array}{l}\text { Criar programa para melhorar a } \\
\text { eficiência da Gestão de }\end{array}$ & $\begin{array}{l}\text { Número de pregões } \\
\text { realizados; } \\
\text { Número de adesão a } \\
\text { atas de registro de } \\
\text { preço; } \\
\text { Número de compras } \\
\text { compartilhadas com } \\
\text { os demais Campi; }\end{array}$ & $\begin{array}{l}\text { Conclusão } \\
\text { até } \\
\text { Dez/2017 }\end{array}$ & $\begin{array}{l}60 \% \text { da } \\
\text { meta }\end{array}$ & $\begin{array}{l}\text { Criação de planilha com toda a movimentação do } \\
\text { processo de compras, disponibilizada em rede, } \\
\text { facilitando a visualização e a busca de informações } \\
\text { por parte dos gestores; } \\
\text { Incentivar as compras compartilhadas entre os Campi, } \\
\text { onde determinado Campus será responsável pela } \\
\text { aquisição de um tipo de material específico, e os } \\
\text { demais adeririam ao pregão, evitando fazer dois } \\
\text { pregões para o mesmo tipo de item em Campi } \\
\text { diferentes. }\end{array}$ \\
\hline
\end{tabular}




\begin{tabular}{|c|c|c|c|c|}
\hline & $\begin{array}{l}\text { Número de dispensas } \\
\text { de licitação } \\
\text { realizadas. }\end{array}$ & & & \\
\hline $\begin{array}{l}\text { Criar critérios de gestão a fim } \\
\text { de agilizar a execução } \\
\text { orçamentária e financeira }\end{array}$ & $\begin{array}{l}\text { Número de critérios } \\
\text { criados; } \\
\text { Número de reuniões } \\
\text { de gestão realizadas. }\end{array}$ & $\begin{array}{l}\text { Conclusão } \\
\text { até } \\
\text { Dez/2017 }\end{array}$ & $\begin{array}{l}60 \% \text { da } \\
\text { meta }\end{array}$ & $\begin{array}{l}\text { Elaboração de planilha de controle mensal de emissão } \\
\text { de empenhos, que possibilita ao final saber quanto } \\
\text { um determinado setor gastou; } \\
\text { Elaboração de planilha para planejar as despesas do } \\
\text { ano seguinte. }\end{array}$ \\
\hline $\begin{array}{l}\text { Criar manuais e fluxos de } \\
\text { trabalho no IFC }\end{array}$ & $\begin{array}{l}\text { Número de manuais } \\
\text { produzidos; } \\
\text { Número de setores } \\
\text { abrangidos. }\end{array}$ & $\begin{array}{l}\text { Conclusão } \\
\text { até } \\
\text { Dez/2017 }\end{array}$ & $\begin{array}{l}40 \% \text { da } \\
\text { meta }\end{array}$ & $\begin{array}{l}\text { Fazer o mapeamento das atividades dos setores; } \\
\text { Criar grupos de trabalho para elaborar os manuais. }\end{array}$ \\
\hline $\begin{array}{l}\text { Criar programa para } \\
\text { diagnosticar, avaliar e otimizar } \\
\text { a distribuição de aulas, } \\
\text { disciplinas e atividades por } \\
\text { área de conhecimento }\end{array}$ & $\begin{array}{l}\text { Percentual de área de } \\
\text { conhecimento } \\
\text { abrangidas. }\end{array}$ & $\begin{array}{l}\text { Conclusão } \\
\text { até } \\
\text { Dez/2017 }\end{array}$ & $\begin{array}{l}50 \% \text { da } \\
\text { meta }\end{array}$ & $\begin{array}{l}\text { Aquisição de Sistema Integrado de Gestão Acadêmica } \\
\text { que irá fornecer informações para as otimizações; } \\
\text { Cadastro dos dados no novo sistema; } \\
\text { Distribuição de disciplinas por área a fim, e não por } \\
\text { curso. }\end{array}$ \\
\hline $\begin{array}{l}\text { Desenvolver mecanismos para } \\
\text { melhorar a distribuição da } \\
\text { carga horária de professores } \\
\text { entre ensino, pesquisa e } \\
\text { extensão }\end{array}$ & $\begin{array}{l}\text { Número de } \\
\text { mecanismos criados; } \\
\text { Percentual de } \\
\text { professores } \\
\text { abrangidos. }\end{array}$ & $\begin{array}{l}\text { Conclusão } \\
\text { até } \\
\text { Dez/2017 }\end{array}$ & $\begin{array}{l}50 \% \text { da } \\
\text { meta }\end{array}$ & $\begin{array}{l}\text { Reformulação da normativa docente que regulamenta } \\
\text { a distribuição entre aula, pesquisa e extensão; } \\
\text { Criação de regulamentação específica para área de } \\
\text { pesquisa; } \\
\text { Criação de regulamentação específica para área de } \\
\text { extensão. }\end{array}$ \\
\hline $\begin{array}{l}\text { Instituir política de } \\
\text { comunicação }\end{array}$ & $\begin{array}{l}\text { Número de } \\
\text { solicitações on-line; } \\
\text { Número de políticas } \\
\text { criadas. }\end{array}$ & $\begin{array}{l}\text { Manter o } \\
\text { resultado de } \\
2015\end{array}$ & $100 \%$ & $\begin{array}{l}\text { Revisão frequente das ferramentas criadas no site, } \\
\text { para que todas as solicitações sejam feitas on-line; } \\
\text { Revisão frequente das normativas criadas para } \\
\text { padronizar o uso da "marca IFC" em redes sociais e } \\
\text { assinatura de e-mails institucionais. }\end{array}$ \\
\hline $\begin{array}{l}\text { Implantar e consolidar as } \\
\text { Coordenações de } \\
\text { Comunicação, normatizando e } \\
\text { formando equipes como } \\
\text { profissionais da área }\end{array}$ & $\begin{array}{l}\text { Número de } \\
\text { normativas emitidas. }\end{array}$ & $\begin{array}{l}\text { Manter o } \\
\text { resultado de } \\
2015\end{array}$ & $100 \%$ & $\begin{array}{l}\text { Trabalho em conjunto com as coordenações de } \\
\text { comunicação dos demais Campi para padronizar as } \\
\text { ações. }\end{array}$ \\
\hline
\end{tabular}

A elaboração do BSC do IFC Campus Araquari tomou como base o planejamento estratégico desenvolvido pelo IFC para os anos de 2013 a 2017. O mapa estratégico apresentado no PE divide-se em 3 perspectivas: Atuação Institucional, Processos Internos e Recursos. Para a elaboração do painel de desempenho os objetivos estratégicos foram extraídos do mapa apresentado no $\mathrm{PE}$, sendo analisada apenas a perspectiva Processos Internos, e os indicadores estratégicos elaborados por meio de entrevistas com os gestores.

Frente aos trabalhos similares encontrados, percebe-se que objetivos estratégicos e alguns indicadores da perspectiva processos internos do IFC relacionados a políticas de comunicação estão alinhados com os apresentados nos estudos anteriores em instituições públicas de ensino. Em seu estudo sobre a proposição de um mapa estratégico na forma do BSC para uma universidade estadual do Paraná, Martins (2015) descreve que a perspectiva processos internos apresentou os seguintes objetivos e indicadores estratégicos: Melhoria dos Indicadores de notas da CAPES: Indicadores de notas CAPES por programa de pós-graduação; Comunicação, Adequação e avaliação contínua institucional: Ouvidoria para docentes, discentes e agentes universitários.

Não obstante, tem-se ainda: Viabilidade de ampliação de vagas nos cursos existentes, Viabilidade de novos cursos nos campis e Revisão das grades dos cursos de graduação; e Incentivo a servidores e docentes: Percepção dos incentivos aos servidores. No estudo realizado por Farias et al. (2012), que propuseram de um mapa estratégico como ferramenta de comunicação da estratégia em uma Instituição de Ensino Público Federal de Santa Catarina, a perspectiva processos internos também apresentou 4 objetivos estratégicos, que são apresentados a seguir com seus respectivos indicadores: Ampliar e manter melhorias nos campi: 
Cronograma físico/financeiro das obras/licitações realizadas e empresas contratadas, Execução das obras conforme cronograma físico/financeiro.

Em continuidade, tem-se: Melhorar o fluxo informacional do IF-SC: Índice de satisfação dos usuários internos em relação ao site e à intranet, Percentual de servidores capacitados; Desenvolver e implantar os sistemas gerenciais: Percentual de sistemas implantados; e Estruturar a diretoria de administração e descentralizar as atividades: Número de setores implantados, número de atividades executadas nos campi.

Não foram identificados estudos anteriores que elaboraram o painel de desempenho em instituições de ensino. Com a elaboração do painel, é possível verificar a situação atual do desempenho da instituição, em relação aos objetivos estratégicos pré-determinados no mapa. Por meio do painel os gestores poderão fazer o acompanhamento dos objetivos, fazendo ajustes nas iniciativas caso as metas não estejam sendo atingidas. Com isso, considera-se que este trabalho avançou em relação ao tema de BSC em instituições de ensino.

\section{CONCLUSÕES}

Planejar estrategicamente objetiva direcionar os rumos da instituição e dar a ela sustentabilidade apontando para respostas de questões fundamentais como: Onde estamos? Onde queremos chegar?. Entretanto, os resultados serão mais factíveis na medida em que o processo tenha monitoramento contínuo e integrado, utilizando-se a legitimação constante dos indicadores, das metas e da relevância atribuída a cada um deles. O sucesso do Planejamento depende da capacidade de execução do que foi planejado, e deve contar com o comprometimento de todos.

Deste modo, o gestor deve se valer de ferramentas para avaliar o desempenho da instituição e, dentre os vários métodos de avaliação, destaca-se o Balanced Scorecard (BSC), desenvolvido no início da década de 1990 por Kaplan e Norton, que utilizaram os indicadores de desempenho para informar os motivos de sucesso atual e futuro da empresa a fim de alcançar as metas traçadas.

O BSC é mais comumente utilizado em empresas privadas, no entanto, este método de avaliação de desempenho também pode ser aplicado em outras áreas, como instituições de ensino públicas ou privadas, como pode ser visto neste artigo que demonstra a implementação do BSC em um Instituto Federal de Ensino. Por meio do estudo, observou-se também que o BSC pode ser considerado como uma promissora ferramenta para a avaliação de desempenho de instituições de ensino.

Para a implementação do BSC na instituição em estudo, partiu-se do mapa estratégico extraído do Planejamento Estratégico 2013-2017 da instituição. Após entrevistas com gestores, definiu-se os indicadores, as metas, a situação atual e as iniciativas, possibilitando assim, a construção do painel de desempenho do Balanced Scorecard. Por meio das entrevistas, foi possível constatar que a estratégia não foi amplamente divulgada para os Campi pela Reitoria e que não estão sendo elaborados anualmente os planejamentos táticos e estratégicos, conforme sugerido no PE.

Esta pesquisa limitou-se a analisar a perspectiva de Processos Internos - Eficiência Operacional, no âmbito do Instituto Federal Catarinense, Campus Araquari. Com a elaboração do painel de desempenho, 
constatou-se que a maior parte dos objetivos já alcançou $50 \%$ ou mais da meta, destacando-se apenas o objetivo de criar manuais e fluxos de trabalho no IFC, que atingiu somente $40 \%$.

Desta forma, o objetivo do estudo foi alcançado ao descrever as etapas para a aplicação do Balanced Scorecard no Instituto Federal Catarinense. No campo teórico este artigo contribui para o desenvolvimento de outros estudos na área de avaliação de desempenho, e pode ser aplicado nas outras perspectivas do mapa estratégico, em outros Campi do IFC ou em demais instituições de ensino.

\section{REFERÊNCIAS}

AL-HOSAINI, F. F.; SOFIAN, S.. A review of Balanced Scorecard framework in higher education institution (HEls). International Review of Management and Marketing, v.5, n.1, p.26-35, 2015.

AMARATUNGA, D.; BALDRY, D.; SARSHAR, M.. Process improvement through performance measurement: the Balanced Scorecard methodology. Work study, v.50, n.5, p.179-189, 2001. DOI:

https://doi.org/10.1108/EUM0000000005677

BEUREN, I. M.. Como elaborar trabalhos monográficos em contabilidade: Teoria e Prática. 3 ed. São Paulo: Atlas, 2006.

BRASIL. Lei n.11.892, de 29 de dezembro de 2008. Institui a Rede Federal de Educação Profissional, Científica e

Tecnológica, cria os Institutos Federais de Educação Ciência e Tecnologia, e dá outras providências. Brasília: DOU, 2008.

BRASIL. Ministério da Educação. Expansão da Rede Federal de Educação Profissional, Científica e Tecnológica. Brasília: ME, 2016.

COSTA, R. C.; SCARE, R. F.. Balanced Scorecard na Gestão de uma Organização Estudantil Universitária: o estudo de caso AIESEC. In: ENCONTRO DA ASSOCIAÇÃO NACIONAL DE PÓSGRADUAÇÃO E PESQUISA EM ADMINISTRAÇÃO. Anais. Rio de Janeiro: ANPAD, 2007.

DALFOVO, O.; LIMA, M. P.. Estudo para implantação do Balanced Scorecard em uma IES como um observatório da educação. Revista de Contabilidade do Mestrado em Ciências Contábeis da UERJ, v.14, n.3, p.60-77, 2009.

ENSSLIN, L., ENSSLIN, S. R.. Material didático apresentado na Disciplina: Avaliação de Desempenho do Programa de Pós-graduação em Engenharia de Produção da Universidade Federal de Santa Catarina. Florianópolis: UFSC, 2007.

FARIAS, S.; NUNES, G. S. F.; LYRIO, M.; LUNKES, R. J.. O desenvolvimento de um mapa estratégico como ferramenta para comunicação da estratégia Pública Federal de Ensino de Santa Catarina. In:CONGRESSO USP DE CONTROLADORIA E CONTABILIDADE. Anais. São Paul: USP, 2012.

FERREIRA, H.; CASSIOLATO, M.; GONZALEZ, R.. Uma experiência de desenvolvimento metodológico para avaliação de programas: o modelo lógico do programa segundo tempo. Brasília: IPEA, 2009.

GAUTREAU, A; KLEINER, B. H.. Recent trends in performance measurement systems: the Balanced Scorecard approach.
Management Research News, v.24, n.4, p.153-156, 2001. DOI: https://doi.org/10.1108/01409170110782793

GIACOBBO, M.. O desafio da implementação do planejamento estratégico nas organizações públicas. Revista do TCU, Brasília, v.28, n.74, 1997.

GRAY, D. E.. Pesquisa no mundo real. Porto Alegre: Artmed, 2012.

HITT, M. A.; IRELAND, R. D.; HOSKISSON, R. E. Administração estratégica. São Paulo: Bookman, 2003.

HLADCHENKO, M.. Balanced Scorecard: a strategic management system of the higher education institution. International Journal of Educational Management, v.29, n.2, p.167-176, 2015. DOI: https://doi.org/10.1108/IJEM-11-2013-0164

IFC. Instituto Federal Catarinense. Planejamento Estratégico 2013-2017. Blumenau: IFC, 2014.

KAPLAN, R.; NORTON, D.. A Estratégia em Ação: Balanced Scorecard. 14 ed. Rio de Janeiro: Campus, 1997.

KAPLAN, R.S.; NORTON, D. P.. The Balanced Scorecard measures that drive performance. Harvard Business Review, v.70, n.1, p.71-79, 1992.

LIMA, E. P.; COSTA, S. E. G.; ANGELIS, J. J.. Strategic performance measurement systems: a discussion about their roles. Measuring Business Excellence, v.13, n.3, p.3948, 2009. DOI: https://doi.org/10.1108/13683040910984310

MCADAM, R.; BAILIE, B.. Business performance measures and alignment impact on strategy: the role of business improvement models. International Journal of Operations \& Production Management, v.22, n.9, p.972-996, 2002. DOI: https://doi.org/10.1108/01443570210440492

MOORAJ, S.; OYON, D.; HOSTETTLER, D.. The Balanced Scorecard: a necessary good or an unnecessary evil?. European Management Journal, v.17, n.5, p.481-491, 1999. DOI: https://doi.org/10.1016/S0263-2373(99)00034-1

NEELY A. The evolution of performance measurement research: developments in the last decade and a research agenda for the next. International Journal of Operations \& Production Manager, v.25, n.12, p.1264-1277, 2005. DOI: https://doi.org/10.1108/01443570510633648

PAPENHAUSEN, C.; EINSTEIN, W.. Implementing the Balanced Scorecard at a college of business. Measuring 
Business Excellence, v.10, n.3, p.15-22, 2006. DOI: https://doi.org/10.1108/13683040610685757

PEREIRA, S. C. S.. O planejamento estratégico em organizações públicas: um estudo de caso das organizações militares prestadoras de serviço. In: CONGRESO INTERNACIONAL DEL CLAD SOBRE LA REFORMA DEL ESTADO Y DE LA ADMINISTRACIÓN PÚBLICA, 11. Anais. Ciudad de Guatemala: CLAD, 2006.

PHILBIN, S. P.. Design and implementation of the Balanced Scorecard at a university institute. Measuring Business Excellence, v.15, n.3, p.34-45, 2011. DOI: https://doi.org/10.1108/13683041111161148

PU゚ČEK, M.; ŠPAČEK, D.. Strategic Public ManagementSelected Experiences with BSC Implementation. Transylvanian Review of Administrative Sciences, v.10, n.41, p.146-169, 2014.

ROSA, M. M.; PETRI, S. M.; BERNARDO, F. D.; VISANI JUNIOR, C.; BIANCO, P.. Alinhamento das estratégias de uma entidade pública: um estudo de caso. Revista de Gestão e Contabilidade da UFPI, v.1, n.2, p.78-92, 2014.

SAYED, N.. Ratify, reject or revise: Balanced Scorecard and universities. International Journal of Educational Management, v.27, n.3, p.203-220, 2013. DOI: https://doi.org/10.1108/09513541311306440

SCHOBEL, K.; SCHOLEY, C.. Balanced Scorecards in education: focusing on financial strategies. Measuring Business Excellence, v.16, n.3, p.17-28, 2012. DOI: https://doi.org/10.1108/13683041211257385

TAPINOS, E.; DYSON, R. G.; MEADOWS, M.. The impact of performance measurement in strategic planning. International Journal of Productivity and Performance Management, v.54, n.6, p.370-384, 2005. DOI: https://doi.org/10.1108/17410400510604539

UCHOA, C. E.. Elaboração de indicadores de desempenho institucional. Brasília: ENAP, 2013.
ULYSSEA, D. S.; MARTINS, C.; SILVA, J. D.; GIAROLA, P. G.; LIMA, C. R. M.. Balanced Scorecard em uma instituição de ensino superior: uma proposta de modelo para gestão estratégica. Revista Catarinense da Ciência Contábil, v.12, n.35, p.28-47, 2013. DOI: http://doi.org/10.16930/2237$\underline{7662 / \text { rccc.v12n3 }}$

UMASHANKAR, V.; DUTTA, K.. Balanced Scorecards in managing higher education institutions: an Indian perspective. International Journal of Educational Management, v.21, n.1, p.54-67, 2007. DOI: https://doi.org/10.1108/09513540710716821

WALL, F.; GREILING, D.. Accounting information for managerial decision-making in shareholder management versus stakeholder management. Review of Managerial Science, v.5, n.3, p.91-135, 2011. DOI: https://doi.org/10.1007/s11846-011-0063-8

WAWERU, N.; PORPORATO, M.; HOQUE, Z.. Performance Measurement Practices in Canadian Government Departments: A Survey. Wasington: MAS Meeting Paper, 2007. DOI: https://doi.org/10.1504/IJAAPE.2008.020849

WICKRAMASINGHE, D.; GOONERATNE, T.; JAYAKODY, J. A. S. K.. Interest lost: the rise and fall of a Balanced Scorecard project in Sri Lanka. Bingley: Emerald Group Publishing Limited, 2007.

WU, H. Y.; LIN, Y. K.; CHANG, C. H.. Performance evaluation of extension education centers in universities based on the Balanced Scorecard. Evaluation and Program Planning, v.34, n.1, p.37-50, 2011. DOI: https://doi.org/10.1016/i.evalprogplan.2010.06.001

YU, M. L.; HAMID, S.; IJAB, M. T.. The e-Balanced Scorecard (e-BSC) for measuring academic staff performance excellence. Higher Education, v.57, n.6, p.813-828, 2009. DOI: https://doi.org/10.1007/s10734-009-9197-x

ZANGOUEINEZHAD, A.; MOSHABAKI, A.. Measuring university performance using a knowledge-based Balanced Scorecard. International Journal of Productivity and Performance Management, v.60, n.8, p.824-843, 2011. DOI: https://doi.org/10.1108/17410401111182215 